\title{
Mechanochemistry and the Other Branches of Chemistry: Similarities and Differences
}

\author{
L. TAKACS* \\ Department of Physics, University of Maryland Baltimore County \\ 1000 Hilltop Circle, Baltimore MD 21250, USA \\ (Received September 28, 2011)
}

\begin{abstract}
It is argued that mechanical action can induce a unique chemical reaction, if enough mechanical energy is concentrated in the bonds involved in the process to bypass the activation energy. This can happen at crack tips, at the core of dislocations, or at the asperities of colliding or sliding surfaces. A mechanical reaction is always complex, as the macroscopic work is distributed among many possible reaction sites. In comparison, an elementary photochemical reaction is induced by a single photon, while thermochemical reactions rely on the accidental concentration of energy by thermal fluctuations. The paper also compares mechanochemical synthesis in a ball mill with reactions under well-defined loading conditions and mechanochemical experiments carried out on the molecular scale. Closer interaction among those branches of mechanochemistry is urged.
\end{abstract}

PACS: 81.20.Ev, 82.33.Pt, 82.30.-b

\section{Introduction}

Mechanical action induces chemical change in a variety of situations, either on purpose or unintentionally. For example, ball milling activates the reactants and initiates or promotes chemical reactions during mechanosynthesis [1]. Applied to metallurgical systems, the same process is called mechanical alloying [2]. Elastic deformation influences chemical behavior, e.g. decreases the resistance to corrosion [3]. In a sliding contact, chemical changes happen to both the sliding surfaces and the lubricant [4]. Phase transformations and reactions can occur due to plastic deformation, as shown in high pressure - torsion experiments [5]. It is possible to form and break bonds in individual molecules, using atomic force microscopy (AFM) [6]. Muscles convert chemical energy to motion [7]. Even if the last example is left for biochemistry, we are dealing with a broad variety of phenomena. Should we consider all of them "mechanochemistry"? What are the criteria? What makes mechanochemical reactions unique?

Although chemical changes caused by mechanical action have been known since the beginnings of chemistry [8], the distinct difference between thermochemical and mechanochemical reactions was demonstrated only at the end of the 19th century, when M. Carey Lea showed that $\mathrm{AgCl}$ and $\mathrm{HgCl}_{2}$ decompose upon tritura-

* e-mail: takacs@umbc.edu tion, although they melt or sublime when heated [9]. These results prompted Wilhelm Ostwald to consider mechanochemistry a separate branch of chemistry [10]. Starting from his classification, it is natural to identify the distinguishing features of mechanochemistry by comparing them with the other areas of chemistry, namely thermochemistry, photochemistry, and electrochemistry.

\section{Comparison of reactions according to the source of energy}

Any chemical reaction must pass through an energy barrier, thus its rate relates to the Arrhenius equation

$$
k(T)=A \exp \left(-\frac{E_{\mathrm{a}}}{k_{\mathrm{B}} T}\right) .
$$

The activation energy $E_{\mathrm{a}}$ is the energy needed to induce an elementary reaction.

The energy source of ordinary thermochemical reactions is heat. In order to initiate the reaction, thermal fluctuations must concentrate sufficient energy in the bonds involved in the process to bypass the activation energy. As typically $E_{\mathrm{a}} \gg k_{\mathrm{B}} T$, this does not happen very easily, limiting the rate of thermochemical reactions.

Photochemical reactions are initiated by the absorption of light. Each photon delivers an $h \nu$ "packet" of energy to an atom or molecule locally. Often $h \nu>E_{\text {a }}$, thus a single photon initiates the reaction in one molecule. There is no need for the accumulation or concentration of energy. 
Electrochemical reactions are more complicated, as the same electron that delivers a batch of energy is also a species of the reaction. But from the energetic point of view, they are similar to photochemical reactions, thus we will not consider them in the forthcoming discussion.

Mechanochemical reactions that differ from their thermal counterparts happen, if enough energy is available locally to bypass the activation energy of an atypical chemical reaction. In ductile bulk materials such concentration of energy takes place when macroscopic plastic deformation creates slip planes and dislocations. Energy is also concentrated close to the tip of a crack or at the asperities of sliding or colliding surfaces.

\section{Single-molecule mechanochemistry}

Mechanochemical reactions in bulk samples are inherently complicated, as they take place at many sites simultaneously. A macroscopic amount of external mechanical work is distributed to a large number of sites and each may be the place of a chemical reaction. In most practical cases, this complexity is unavoidable. For fundamental understanding, atomic-scale experiments that involve only a few atoms or molecules, are extremely valuable.

Single-molecule studies address the most fundamental step of mechanochemical reactions, namely the rupture of a single bond. Investigations are in progress in several directions:

(a) The unfolding, stretching, and rupture of long organic molecules are studied under tension in an AFM [11]. While very interesting and illuminating, such studies are carried out in a solution that often contains active ingredients. Therefore, the exact role of the mechanical pull is not clear.

(b) Forming, stretching, and rupturing of a single-atom-thick wire in high vacuum is possible by pulling a gold-coated AFM tip away from a gold surface [12]. Both experiment and simulation [13] provide about $1.5-$ $1.8 \mathrm{nN}$ for the strength of a single $\mathrm{Au}-\mathrm{Au}$ bond. The simulation also shows that the length of the neighboring bonds increases before a bond breaks, thus a substantial amount of elastic energy builds up.

(c) Breaking of bonds between a substrate and a complex molecule. For example, a simulation of how the bond between a $\mathrm{Cu}$ surface and a $\mathrm{CH}_{3} \mathrm{CH}_{2} \mathrm{~S}$ molecule ruptures reveals substantial difference between the effects of heating and pulling [14].

As a first approximation, breaking of a bond in a single-molecule experiment can be described in terms of a modified Arrhenius equation inspired by the Zhurkov equation proposed to describe time-dependent mechanical phenomena [15]:

$$
k(T)=A \exp \left(-\frac{E_{\mathrm{a}}-\alpha F}{k_{\mathrm{B}} T}\right) .
$$

The term $\alpha F$ was added to the Arrhenius equation to describe the effect of the external tensile force $F$; $\alpha$ is an appropriate constant. The behavior of the molecule or cluster depends on the relative magnitudes of $E_{\mathrm{a}}, \alpha F$, and $k_{\mathrm{B}} T$. If $\alpha F$ is small, the reaction is essentially thermochemical, but the mechanical tension lowers the activation energy needed to break a bond. If the experiment is carried out in a liquid or gaseous medium, $E_{\mathrm{a}}$ incorporates the interaction with the environment in addition to the properties of the investigated molecule. If $\alpha F$ is large, mechanical tension dominates; if $\alpha F>E_{\mathrm{a}}$, the effect is purely mechanical. The two extremes differ more clearly at low temperature.

\section{Mechanochemistry in bulk matter}

If the external stresses do not exceed the elastic limit, any chemical change is essentially thermochemical. The mechanical deformation is distributed among all the bonds of the sample and acts as a perturbation, similar to the case of small $\alpha F$ in a single-molecule experiment. In practice, phase transformations and chemical reactions result in local volume changes and consequently in large local stresses and plastic deformation. This plastic deformation can result in chemical changes, sometimes termed "hidden mechanochemistry" [3]. Corrosion under the influence of elastic stress is a surface phenomenon, thus it is a good example of a purely elastic effect. This case will not be discussed any further.

If the deformation is larger than the elastic limit, the external mechanical work is still distributed among many bonds, but the distribution is far from uniform. Energy is concentrated:

- at the tip of the crack during the fracture of a brittle material,

- in slip planes and within the slip planes in dislocations during plastic deformation of a ductile material,

- at asperities between two surfaces sliding over each other or impacting each other.

In every case, many bonds are affected simultaneously and the distribution of the external work into a multitude of affected sites is an essential component of any mechanochemical phenomenon.

There are two possible ways to study bulk mechanochemical processes:

- Fracture, phase transformations, and reactions are investigated under well-defined loading conditions. Such experiments are intended for fundamental investigations. The objective is to design simple situations that can be investigated both experimentally and theoretically (including computer simulation).

- Mechanochemical synthesis is carried out in milling devices. It is a complex mixture of stochastic processes that is difficult to describe on the fundamental level. On the other hand, it is relatively easy to 
obtain bulk empirical information about the reaction kinetics. Many elementary steps are involved in ball milling and several have mechanochemical elements.

Further progress in mechanochemistry requires closer connection between fundamental studies and practical mechanochemical synthesis.

\section{Reactions and alloying under simple loading conditions}

Breaking of bonds at the tip of a crack during the cleavage of a brittle crystal is a fundamental mechanochemical phenomenon that can be studied under controlled conditions [16, 17]. Usually the emission of light, electrons, atoms, and atom clusters is measured as a function of time. The simple geometry also lends itself to theoretical investigations [18] and computer modeling [19]. The speed of the crack propagation is comparable to the speed of sound in the medium, thus it crosses a single unit cell in less time than the period of a phonon. Consequently, there is no time for thermal equilibration during the passage of the crack, thus the initial breaking of bonds is a direct mechanical effect. As in the case of breaking a single-atom-thick wire, much energy is stored by the elastic deformation of the neighboring bonds. This energy gets distributed and results in local temperature increase and thermally induced effects. As a consequence, both prompt mechanochemical emission associated with the initial passage of the crack and delayed thermal emission are observed. Also, radicals are formed along the freshly created surfaces that result in surface reconstruction and increased reactivity.

Spring studied chemical reactions initiated by the application of high pressure already in the 1880's [20], although it is difficult to separate the effects of hydrostatic compression and secondary shear in his results. The first experiments using a controlled combination of hydrostatic pressure and shear were carried out by Bridgeman [5]. He determined that the kinetics of structural changes scales with the amount of plastic shear strain rather than time, suggesting that the process is driven mechanically rather than thermally. The relative simplicity of the high pressure-torsion experiment allowed the development of a multiscale model that treats both macroscopic material flow and strain-induced nucleation of product phases at the tip of a dislocation pile-up in a coherent fashion [21].

Another relatively simple situation that could be analyzed theoretically is repeated cold rolling or pressing of a multilayer. The experiments of Shingu and Ishihara [22] provided useful empirical information about the role of structure refinement by plastic deformation. However, detailed theoretical analysis and extension of the experiment to brittle or chemically reactive materials has not been attempted yet. This could be a fruitful area for future studies.

\section{Mechanochemistry in a ball mill}

Practical mechanochemical synthesis takes place in the stochastic environment of a high-energy ball mill where various processes take place simultaneously. Although thermal relaxation and thermally induced reactions certainly play a significant role, direct mechanochemical effects are also clearly present.

The vigorous motion of the milling balls carries around the powder particles, facilitating mixing, and the development of a coating layer over the tool surfaces. Although this process seems simple, it is poorly understood. The motion and mixing of powders are essentially mechanical processes, although adhesion and tribocharging also play some role.

The most important component of the activation of powders is particle size reduction. Whether it happens by brittle fracture or as a result of severe plastic deformation, the results are fresh surfaces, broken bonds, and the formation of surface radicals. At least in part, direct mechanical effects are responsible for these processes. Much of the energy consumption of ball mills goes into the creation of fresh surfaces [23].

Repeated plastic deformation during impulsive compression between the milling tools involves slip, mediated by the creation and moving of dislocations. Energy is concentrated around dislocation pile-ups, creating chemically active centers. These are essentially mechanochemical processes, not unlike the ones encountered in a high pressure-torsion experiment. Most of the energy provided by a ball mill is used to create fresh surfaces and lattice defects. In some alloy systems, such as $\mathrm{Ni}_{40} \mathrm{Ti}_{60}$, the accumulation of defects results in amorphization. It was shown that the kinetics of amorphization scales with the mechanical dose, defined as the energy absorbed by unit mass of the powder [24]. Mechanical dose is the proper kinetic variable of other mechanochemical processes as well. If a deviation occurs, the typical reason is thermal relaxation, possibly even local melting, if the milling intensity is high, or too much elastic deformation, if the milling intensity is low.

While mechanically created defects are certainly the key to activation, the question remains whether direct mechanochemical reactions are also taking place in ball milling. Probably much of the chemical change is thermally driven, only faster than usual due to the existence of mechanically activated centers and fast diffusion paths along dislocations and grain boundaries. Yet direct mechanochemical reactions are also possible when shearing occurs across interfaces [25] or reactant particles rub against each other and either new compound grains rollers - form [26] or direct mixing takes place at the interface [27].

Although it would be useful to come up with a complete and predictive model of ball milling-induced chemical reactions and mechanical alloying, the process is too complicated and consequently the development of such a model is unlikely. Understanding can be derived from 
highly simplified global models [28] and theories that focus on certain aspects of the process [29]. Inevitably, the inadequacies of models must be filled with well-planned empirical exploration.

\section{Conclusions}

Unique mechanochemical reactions take place, when mechanical energy gets concentrated on the atomic level, so that the locally available energy is comparable to or even larger than the activation energy of a reaction that would be impossible thermally. Macroscopic mechanochemical reactions are inherently complex, as the macroscopic external work gets distributed into many reaction sites, thus processes on the macroscopic and atomic scales take place simultaneously. A mechanochemical reaction is more complicated than a photochemical one, where a single photon initiates the process in a single molecule.

Practical mechanochemical processing uses ball mills to activate powders and to induce chemical reactions. This is a complicated process that includes mechanochemical steps, but they are often concealed by the mechanics of the mill and the inevitable thermal processes. Fundamental knowledge can be obtained more easily from experiments under well-defined loading conditions, such as high pressure-torsion experiments. The investigation of single molecules or clusters of molecules under the action of an external force is also informative. It is important to apply the knowledge acquired from these fundamental investigations to practical mechanochemistry.

\section{References}

[1] Experimental and Theoretical Studies in Modern Mechanochemistry, Eds. F. Delogu, G. Mulas, Transworld Research Network, Trivandrum, Kerala, India 2010.

[2] C. Suryanarayana, Mechanical Alloying and Milling, Marcel Dekker, New York 2004

[3] E.M. Gutman, Mechanochemistry of Materials, Cambridge International Science Publishing, Cambridge 1998.

[4] Z.V. Todres, Organic Mechanochemistry and its Practical Applications, Taylor and Francis, Boca Raton 2006.
[5] P.W. Bridgman, Phys. Rev. 48, 825 (1935).

[6] A.-S. Duwez, S. Cuenot, C. Jerome, S. Gabriel, R. Jerome, S. Rapino, F. Zarbetto, Nature Nanotechnol. 1, 122 (2006).

[7] F.D. Carlson, D.R. Wilkie, Muscle Physiology, Prentice-Hall, Englewood Cliffs, NJ 1974.

[8] L. Takacs, J. Met. 52, 12 (2000).

[9] M.C. Lea, Am. J. Sci. 3rd Series 47, 377 (1894).

[10] W. Ostwald, Handbuch der allgemeinen Chemie, Band I, Academische Verlagsgesellschaft, Leipzig 1919.

[11] M.K. Beyer, H. Clausen-Schaumann, Chem. Rev. 105, 2921 (2005)

[12] G. Rubio-Bollinger, S.R. Bahn, N. Agraït, K.W. Jacobsen, S. Vieira, Phys. Rev. Lett. 87, 026101 (2001).

[13] E.Z. da Silva, A.J.R. da Silva, A. Fazzio, Phys. Rev. Lett. 87, 256102 (2001).

[14] M. Konopka, R. Turansky, J. Reichert, H. Fuchs, D. Marx, I. Stich, Phys. Rev. Lett. 100, 115503 (2008).

[15] S.N. Zhurkov, Int. J. Fracture Mech. 1, 311 (1965).

[16] P.G. Fox, J. Soria-Ruiz, Proc. R. Soc. Lond. A 317, 79 (1970).

[17] F.Kh. Urakaev, Phys. Chem. Minerals 34, 351 (2007).

[18] H.L. Tan, W. Yang, J. Mater. Sci. 31, 2653 (1996).

[19] J.G. Swadener, M.I. Baskes, M. Nastasi, Phys. Rev. Lett. 89, 085503 (2002).

[20] W. Spring, Bull. Soc. Chim. 39, 641 (1883).

[21] V.I. Levitas, Phys. Rev. B 70, 184118 (2004).

[22] P.H. Shingu, K.N. Ishihara, Mater. Trans. JIM 36 , 96 (1995).

[23] P.Yu. Butyagin, A.N. Streletskii, Phys. Solid State 47, 856 (2005)

[24] F. Delogu, L. Schiffini, G. Cocco, Philos. Mag. A 81 , 1917 (2001).

[25] P. Bellon, R.S. Averback, Phys. Rev. Lett. 74, 1819 (1995).

[26] V.V. Zyryanov, Russ. Chem. Rev. 77, 105 (2008).

[27] F. Delogu, G. Cocco, Phys. Rev. B 74, 035406 (2006).

[28] T.H. Courtney, Mater. Trans. JIM 36, 110 (1995).

[29] B.B. Khina, F.H. Froes, J. Met. 48, 36 (1996). 